\title{
Vorkommen von Astropecten aranciacus (Asteroidea, Echinodermata) in Abhängigkeit von Umweltbedingungen
}

\author{
H. Burla, B. Pabst \& W. Stahel \\ Zoologisches Museum der Universität Zürich; \\ Zürich, Schweiz
}

\begin{abstract}
Environmental conditions affecting the occurrence of Astropecten aranciacus (Asteroidea, Echinodermata). In shallow water off the north coast of Sardinia (western Mediterranean) the frequency of occurrence of the starfish Astropecten aranciacus was recorded in 70 sample areas of $25 \mathrm{~m}^{2}$ each. Four environmental factors, namely depth, average sand grain diameter, number of molluse species, and number of individuals of the genus Ecbinocardium proved to be related to the abundance of $A$. aranciacus. The sediment contained many species, each, as a rule, being present at low abundance. The molluscs found and their sizes are listed.
\end{abstract}

\section{EINLEITUNG}

Astropecten aranciacus ist im Mittelmeer die größte Art seiner Gattung, tritt an manchen Küstenstellen reichlich auf und dürtte in seinem Habitat, dem sandigen Infralitoral, als unspezialisierter Räuber einen kontrollierenden Einfluß auf die Bestände seiner Beutetiere ausüben. Doch wissen wir nicht, von welchen Eigenschaften des Habitats $A$. aranciacus im Vorkommen und damit in der Wirkung auf andere Glieder der Lebensgemeinschaft beeinflußt wird.

Prcard (1965) zählt $A$. aranciacus zur SGCF-Biocoenose („sables grossiers et fins graviers sous l'influence des courants de fond"). MAssé (1966) bezeichnet $A$. aranciacus als wenig anspruchsvoll: "L'espèce vit aussi bien sur des graviers mal classés que sur des sables fins vaseux"; er findet sie "surtout dans les fonds meubles qui bordent les herbiers des Posidonies", ober- wie unterhalb solcher Rasen. In der Nahrung richtet sich A. aranciacus nach dem Angebot; sein Magen enthält oft viele Mollusken, besonders junge Muscheln geringer Größe, doch werden auch kleinere Seesterne gefressen (PrCARD, 1965; Massí, 1966; eigene Beobachtungen).

A. aranciacus wurde geborgen aus 5 bis $100 \mathrm{~m}$ Tiefe (KoemLer, 1924; CherbonNIER, 1958). Man kennt die Art von verschiedenen Küstenstellen des Mittelmeers, ferner von Gebieten des Atlantik: Portugal, den Kanarischen Inseln, Madeira, Angola (Kofhler, 1921; Cherbonnier, 1958; Tortonese, 1965). Im Nordosten von Sardi- 
nien, wo wir seit einigen Jahren die Okologie von Seesternen in ihrem Habitat untersuchen, trafen wir $A$. aranciacus an allen unseren Arbeitsplätzen an, vor allem in der Abend- und Morgendämmerung sowie nachts (Burla et al., 1972). Doch kennen wir andere Stellen an der Küste von Südfrankreich, Korsika und Sardinien, wo A, aranciacus auch auf Sandgrund selten ist oder fehlt.

Im Sommer 1973 führten wir in zwei benachbarten Buchten stichprobenweise eine Bestandsaufnahme durch. Das Ziel war die Ermittlung von Bedingungen, die auf Vorkommen und Abundanz von $A$. aranciacus Einfluß haben.

\section{METHODEN}

\section{Ort und Datenerhebung}

Abbildung 1 zeigt das Untersuchungsgebiet: zwei Buchten im Golf von Arzachena, die nördliche mit sieben, die südlich anschließende mit elf Sammelstellen. An jeder Stelle wurden vier quadratische Stichprobenfelder von $5 \mathrm{~m}$ Seitenlänge mittels Nylonschnur umgrenzt; als Ausnahme wurden an einer Stelle nur zwei Stichprobenfelder angelegt. In der Zeit vom 23. August bis 18 . September wurden 70 Stichprobenfelder bearbeitet. Thre gesamte Fläche betrug $1750 \mathrm{~m}^{2}$. Auf jedem Stichprobenfeld wurden folgende Arbeiten ausgeführt: Die auf dem Feld sichtbaren Tiere wurden gezählt, zum Teil eingesammelt. Die allgemeinen Bedingungen, wie Tageszeit, Tiefe, Vegetation, wurden notiert. An zwei diagonal gegenüberliegenden Ecken, je $50 \mathrm{~cm}$ außerhalb des Feldes, wurden mittels eines Plastikrohres von $30 \mathrm{~mm}$ Durchmesser Sandproben von $10 \mathrm{~cm}$ Höhe herausgestochen und zur Analyse an Land gebracht; alle nachfolgenden Angaben über Sandqualitäten beziehen sich auf diese ingesamt 140 Proben. An den zwei anderen Ecken, ebenfalls außerhalb des Feldes, wurde ein kurzer Zylinder aus PVC, der ein achtel Quadratmeter Fläche einschließt, $10 \mathrm{~cm}$ tief in den Boden getrieben; der vom Zylinder eingeschlossene Inhalt von 12,5 Liter wurde mittels einer Suceuse aufgesaugt und an Ort und Stelle durch ein Gitter von $2 \mathrm{~mm}$ Maschenweite gesiebt. Der Rückstand wurde zur Oberfläche und ans Ufer gebracht und dort nach Tieren durchsucht. Auf diese Weise wurden 1750 Liter Sand erfaßt.

Mit Hilfe zweier Handrechen aus Eisen, deren Zinken $15 \mathrm{~cm}$ lang sind und etwa $10 \mathrm{~cm}$ tief in den Boden graben, wurde jedes Stichprobenfeld durchpflügt auf der Suche nach Seesternen, die im Sand verborgen liegen.

Die Sandproben wurden am Ufer mit Formalin versetzt und gelangten naß nach Zürich, wo sie analysiert wurden. Fraktioniertes Sieben ergab die Häufigkeitsverteilung der Korndurchmesser nach sieben Größenklassen entsprechend den Maschenweiten $0,063,0,125,0,25,0,5,2,1$ und $4 \mathrm{~mm}$. Aus der Verteilung bestimmten wir die häufigste Klasse, Variationsbreite (Anzahl Klassen mit Häufigkeit $\mathrm{h} \geqq 5 \%$ ), Homogenität (Anzahl Klassen mit $\mathrm{h} \geqq 10 \%$ ) und Standardabweichung. Schließlich wurden die prozentualen Gewichtsanteile von Kalk und organischen Stoffen gemessen.

Nach der Ausdehnung des Posidonienrasens, der auf Fotos aus der Luft erkennbar war, ließ sich für jede Sandfläche, auf der Stichprobenfelder lagen, die „Flächengliederung" klassifizieren nach „offen" (keine Posidonien im weiteren Umkreis), „gegliedert" 
(offene Sandflächen und Posidonien gemischt) und "geschlossen" (kleine Sandflächen umschlossen von Posidonienrasen). Die drei Zustände ergaben im dummy-System (Surrs, 1957) zwei Variable, aus denen sich der Einfluß aller drei Zustände ausrechnen läßt. Dummy-Systeme wurden auch verwendet für die 26 Kalendertage, 4 Tageszeiten (9-12, 12-15, 15-18, 18-21 Uhr) und 18 Tauchstellen.

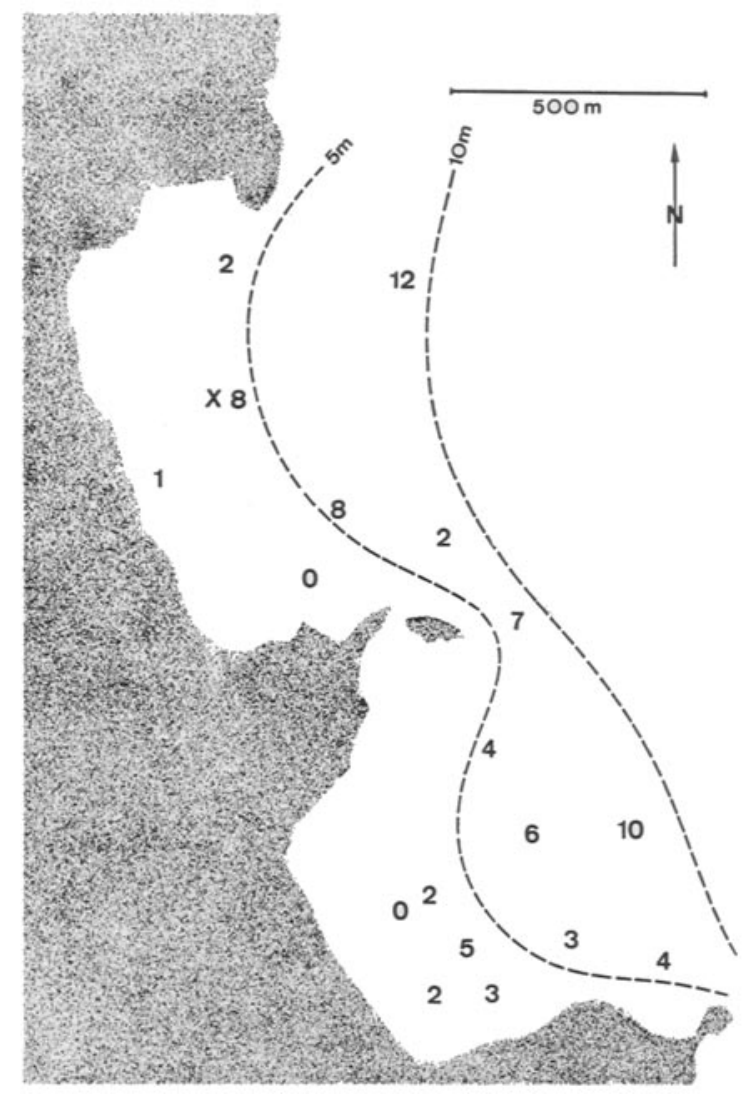

Abb. 1: Die beiden Buchten im Golf von Arzachena (nordöstliches Sardinien), in denen die Arbeit ausgeführt wurde. Die Nummern geben die Anzahl Individuen von Astropecten aranciacus an, die auf je 4 Stichprobenfeldern zu $25 \mathrm{~m}^{2}$ an 18 Stellen gefunden wurden. An der mit $\mathrm{X}$ bezeichneten Stelle wurde bereits 1971 (BuRLa et al., 1972) die Dichte bestimmt

\section{AusschluB von Variabeln}

Die Rohdaten, an denen die dummy-Systeme einen großen Anteil haben, wurden mittels Programm BMDO2R (stepwise multiple regression) provisorisch beurteilt im Hinblick auf Variable, die auf die Abundanz von A. aranciacus einen Einfluß auszuüben scheinen. Beiträge zum $\mathrm{R}^{2}$ von mehr als $1 \%$ lieferten die Tiefe, $8 \mathrm{Kalendertage,}$ 
4 Tauchstellen, 3 Sandqualitäten sowie die Abundanzen von Condylactis, Einsiedlerkrebsen und übrigen Krebsen. Da weder Tage noch Tauchstellen zufällig oder systematisch variiert worden waren und wir annehmen mußten, diese Variabeln maskierten andere, biologisch interessantere Effekte, wurden sie trotz ihres gesamthaft hohen Beitrags zum $\mathrm{R}^{2}$ aus der weiteren Betrachtung eliminiert. Nach ihrem Ausschluß blieben 24 Variable. Für jede Kombination von zwei Variabeln wurde der Korrelationskoeffizient (r) berechnet. Daraufhin wurde eine zweidimensionale Skizze erstellt, in der Variable als Punkte erscheinen und kurze Abstände ein großes $r$ bedeuten. In dieser Darstellung fiel auf, daß alle anorganischen Umweltfaktoren auf eine Seite zu liegen kamen, alle Anzahlen von 'Tieren auf die andere Seite, was bedeutet, daß gesamthaft die Abundanzen der Tierarten nur wenig von den anorganischen Bedingungen abhängen.

Um die Einengung auf wesentliche unabhängige Faktoren weiterzutreiben, wurden alle Variablen ausgeschieden, die mit weniger als $x=0,10$ mit $A$. aranciacus korreliert waren. Diesem Ausscheidungsprozeß fielen zum Opfer: Tageszeiten, Buchten, Vorkommen von Zostera, Condylactis und Muscheln, schließlich zwei Sandqualitäten. Ubrig blieben 15 Variable, die in die Tabellen 1 und 2 eingingen. Wiederholte Regres-

Tabelle 1

Liste der Variabeln, die in die definitive Regressionsrechnung eingingen $\mathrm{n}=$ Gesamtzahl

\begin{tabular}{|clc|}
\hline Nummer & \multicolumn{1}{c|}{ Variable } & $\mathrm{n}$ \\
\hline 1 & Anzahl A. aranciacus-Individuen & 77 \\
2 & Anzahl Mollusken-Arten & 30 \\
3 & Anzahl Echinocardium-Individuen & 32 \\
4 & Anzahl Schnecken-Individuen & 51 \\
5 & Anzahl Sipunculida-Individuen & 11 \\
6 & Anzahl andere Würmer, Individuen & 403 \\
7 & Anzahl Einsiedlerkrebs-Individuen & 38 \\
8 & Anzahl Amphipoda und Isopoda, Individuen & 12 \\
9 & Tiefe & \\
10 & Sandkorndurchmesser: häufigste Klasse & \\
11 & Sandkorndurchmesser: Standard-Abweichung & \\
12 & Sand:0\% Kalk & \\
13 & Sand:\%\% organisches Material & \\
14 & Flächengliederung "offen“" & \\
15 & Flächengliederung "geschlossen" & \\
\hline
\end{tabular}

sionsrechnungen und eine restriktivere Auswahl nach Koorelationskoeffizient führten schließlich zu dem in Tabelle 4 enthaltenen Ergebnis.

Die Variation, die durch die Regression der Tabelle 4 (links, "ungewichtet") erklärt wird, erscheint mit $33 \%$ enttäuschend klein. Es zeigt sich aber, daß der verbleibende Anteil durch die Art der Stichprobennahme bedingt ist. Wir gehen von der Annahme aus, daß die Anzahl Seesterne auf jeder Probefläche einer Poissonverteilung entstammt, deren Mittelwert durch die örtlichen Verhältnisse gegeben ist und mit der Regression geschätzt wird. $\mathrm{Da}$ die Annahme gleicher Varianzen verletzt ist, ist die 
Vorkommen von Astropecten aranciacus

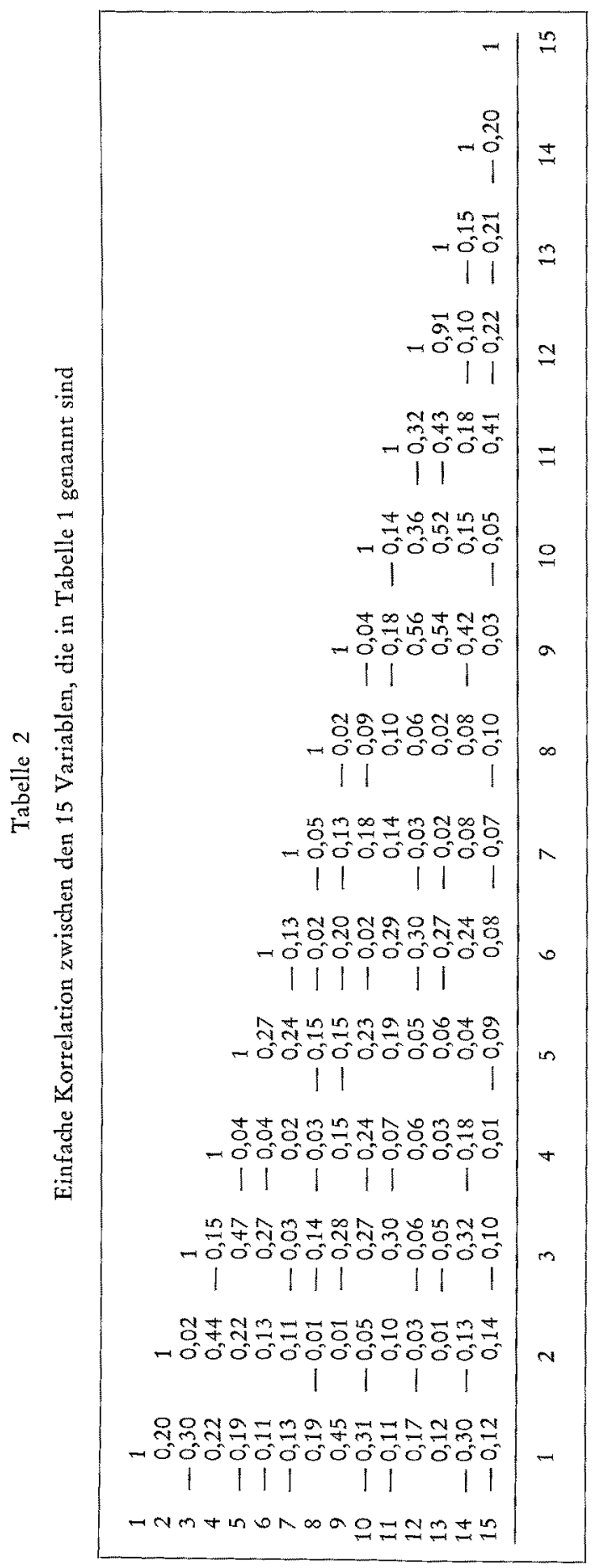


Tabelle 3

Anzahl Stichprobenfelder (f) von $25 \mathrm{~m}^{2}$ Größe, auf denen $\mathrm{i}=0,1,2, \ldots, 5$ Individuen von Astropecten aranciacus gefunden wurden

\begin{tabular}{|crrrrrr|}
\hline $\mathrm{i}$ & 0 & 1 & 2 & 3 & 4 & 5 \\
\hline$f$ & 28 & 22 & 11 & 4 & 4 & 1 \\
\hline
\end{tabular}

zuerst berechnete Kleinste-Quadrate-Lösung nicht optimal. Sie wird verbessert, indem die Beobachtungen gewichtet werden (Draper \& SMITH, 1966; pp. 77-81). Die Gewichte richten sich nach den Varianzen der Beobachtungen, die in unserer Anwendung gleich den zu schätzenden Y-Werten sind. Daraus ergibt sich die Möglichkeit einer schrittweisen Verbesserung einer Schätzung: Mit Gewichten, die aus den Ergebnissen einer ersten Regression - der ungewichteten Kleinste-Quadrate-Lösung - berechnet werden, wird eine gewichtete Regression durchgeführt, aus der sich wieder Gewichte für eine weitere Verbesserung ergeben, und so fort.

Tabelle 4

Ergebnisse der multiplen Regression der Anzahl Seesterne auf vier ausgewählte unabhängige Variable. b: Regressions-Koeffizient; $\mathrm{t}=\mathrm{b} / \mathrm{sb} ; \mathrm{R}^{\mathrm{z}}$ : BestimmtheitsmaB; Zahlen in Klammern: Freiheitsgrade; : Signifikanz fur $\alpha=0,05 ; \%: \alpha=0,01$ bei einfachem Test

\begin{tabular}{|c|c|c|c|c|c|c|}
\hline \multirow{2}{*}{ Unabhängige Variable } & \multirow[b]{2}{*}{ b } & \multicolumn{2}{|c|}{ ungewichtet } & \multirow[b]{2}{*}{$\mathrm{b}$} & gewichtet & \multirow[b]{2}{*}{$\mathrm{R}^{2} \%$} \\
\hline & & $\tau(65)$ & $\mathrm{R}^{2} \% / 0$ & & $t(61)$ & \\
\hline Tiefe & 0,22 & $3,86 \%$ & 20,53 & 0,22 & $4,19 \%$ & 30,1 \\
\hline Sand & 0,282 & $2,35 *$ & 8,40 & 0,256 & $2,81 \div$ & 15,2 \\
\hline Anzahl Molluskenarten & 0,091 & 1,83 & 3,30 & 0,100 & $3,52 *$ & 2,6 \\
\hline Echinocardium-Individuen & $-0,141$ & $-1,09$ & 1,20 & $-0,221$ & $-3,43 *$ & 8,4 \\
\hline Total & - & - & 33,40 & - & - & 56,3 \\
\hline F-Wert & $F(4,65)=$ & & $8,10^{*}$ & $\mathrm{~F}(4,61)=$ & & $19,6 \%$ \\
\hline Ordinatenabschnitt & 0,664 & & & 0,526 & & \\
\hline
\end{tabular}

In Tabelle 4 rechts wurden die Resultate der nach 15 derartigen Iterationsschritten gewonnenen Lösung verglichen mit der ungewichtien Regression. Die erhöhte Signifikanz, insbesondere der Faktoren "Anzahl Molluskenarten" und „Echinocardien-Individuen“, erklären wir uns damit, daß diese Beutetier-Häufigkeiten offenbar vor allem in geringer Tiefe, wo die Seesterne nicht häufig sind, wichtig werden; die Stellen mit geringem Erwartungswert fallen bei der zweiten Art der Regression stärker ins Gewicht. Unter der Annahme von Poisson-Verteilungen ergibt sich die Möglichkeit, die Anpassung mit

$$
\mathrm{X}^{2}=\Sigma\left(\mathrm{Y}_{\mathrm{i}}-\mathrm{Y}_{\mathrm{i}}\right)^{2} / \mathrm{Y}_{\mathrm{i}}
$$

zu prüfen. Da die geschätzten Erwartungen $Y_{i}$ fast zur Hälfte unterhalb 1 liegen, ist die $\chi^{2-V e r t e i l u n g ~ n i c h t ~ a n w e n d b a r . ~ D e r ~ E r w a r t u n g s w e r t ~ b l e i b t ~ g l e i c h ~ d e r ~ Z a h l ~ d e r ~}$ Freiheitsgrade. Nachdem vier Beobachtungen eliminiert waren, für die negative Anzahlen geschätzt wurden, ergab sich ein $\mathrm{X}^{2}$-Wert, der unter dem Erwartungswert lag.

Dieses Ergebnis konnte auf andere Weise bestätigt werden: Aus der Art der 
Datensammlung ergaben sich $\mathrm{p}=31$ Paare von Beobachtungen $\mathrm{Y}_{\mathrm{i} 1}, \mathrm{Y}_{\mathrm{i} 2}$ die an gleichen Tagen im Abstand von etwa 5 Metern gemacht wurden. Die Summe

$$
\sum_{\mathrm{i}=1}^{\mathrm{P}}\left(\mathrm{Y}_{\mathrm{i} 1}-\mathrm{Y}_{\mathrm{i} .}\right)^{2}+\left(\mathrm{Y}_{\mathrm{i} 2}-\mathrm{Y}_{\mathrm{i} .}\right)^{2}=\frac{1}{2} \sum_{\mathrm{i}=1}^{\mathrm{P}}\left(\mathrm{Y}_{\mathrm{i} 1}-\mathrm{Y}_{\mathrm{i} 2}\right)^{2} ; \mathrm{Y}_{\mathrm{i}}=\frac{1}{2}\left(\mathrm{Y}_{\mathrm{i} 1}+\mathrm{Y}_{\mathrm{i} 2}\right)
$$

ergibt eine Quadratsumme zur Schätzung der Residuen-Varianz mit p Freiheitsgraden. Das mittlere Quadrat aus diesen Vergleichen von Nachbarn ergab 1,03, was mit dem entsprechenden Ergebnis 1,09 der Regression gut übereinstimmt.

Die vier ausgewählten Faktoren scheinen also die Variation der Anzahl Seesterne erschöpfend zu erklären.

\section{ERGEBNISSE}

\section{Bestandesdichte von Astropecten aranciacus}

Tabelle 3 veranschaulicht die Häufigkeitsverteilung der Anzahl A. aranciacusIndividuen. Die Summe aller Individuen beträgt 76, der Durchschnitt 1,1 je Stichprobenfeld. Im Mittel entfallen $23 \mathrm{~m}^{2}$ auf einen Seestern. 1971 schätzten wir für die mit $\mathrm{X}$ bezeichnete Stelle in Abbildung 1 eine Bestandesdichte von einem Seestern auf $10 \mathrm{~m}^{2}$. 1973 fanden wir an der gleichen Stelle 8 Seesterne auf $100 \mathrm{~m}^{2}$ Fläche oder 1 Tier auf $12,5 \mathrm{~m}^{2}$, was gut mit der früheren Schätzung übereinstimmt.

Beide Buchten ergaben ähnliche Bestandesdichten. In der nördlichen Bucht wurden 33 Seesterne auf 28 Stichprobenfeldern gezählt (1 Tier pro $21 \mathrm{~m}^{2}$ ), in der südlichen waren es 43 Seesterne auf 42 Feldern (1 Tier pro $24 \mathrm{~m}^{2}$ ).

Die größte Bestandesdichte betrug 12 Seesterne auf $100 \mathrm{~m}^{2}$. Es waren durchwegs mittelgroße Tiere mit einem Radius $\mathrm{R} z$ wischen 7 und $15 \mathrm{~cm}$.

Außer $A$. aranciacus wurden noch 4 Individuen von Astropecten bispinosus gezählt. Astropecten jonstoni, Astropecten platyacantbus und Astropecten irregularis, ebenfalls in den Buchten nachgewiesen, wurden auf den Stichprobenfeldern nicht gefunden. Die bearbeiteten Sandflächen sind also in bezug auf die Gattung Astropecten insoweit einheitlich, als durchwegs die Art A. aranciacus dominiert.

\section{Auf Astropecten aranciacus wirkende Umweltfaktoren}

Tabelle 4 enthält das Ergebnis der Regressionsrechnung. Die Anzahl A. aranciacus-Individuen wird von allen vier Variabeln gesichert beeinflußt.

Die einfache lineare Regression von $A$. aranciacus auf die Tiefe zeigt Abbildung 2a: Je größer die Tiefe, um so mehr Seesterne treten auf.

In Tabelle 4 ist mit „Sand ${ }^{\text {c }}$ die häufigste Klasse in der Häufigkeitsverteilung der Sandkorndurchmesser gemeint. Wie Abbildung $2 \mathrm{~b}$ veranschaulicht, ist die Beziehung positiv: Je gröber der Sand, um so mehr Seesterne. Der Befund deckt sich mit Erfahrungen, die wir bei anderen Gelegenheiten gewannen: (a) A. aranciacus war auf einer mittleren Sandkorngröße am abundantesten (RIBI et al., im Druck); (b) allgemein fan- 

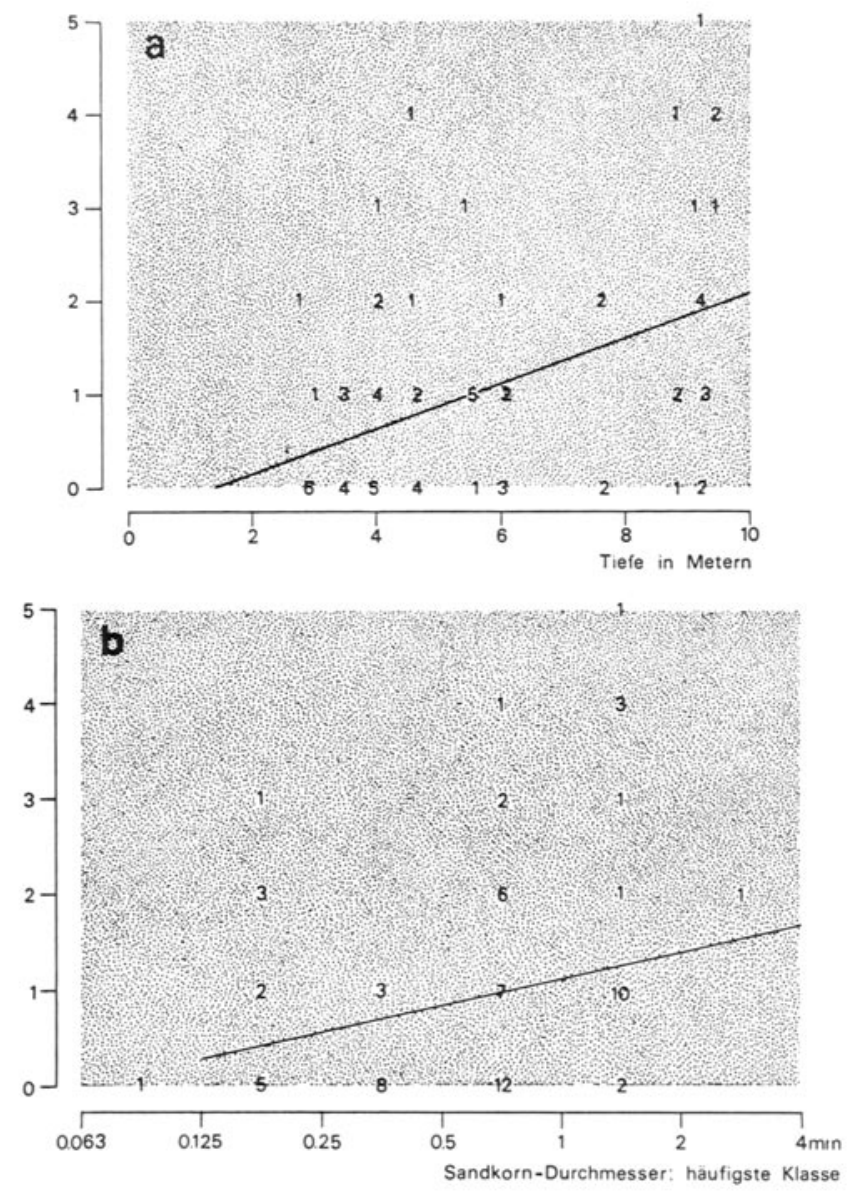

Abb. $2 \mathrm{a}$ und $\mathrm{b}$ : Einfache lineare Regression der Individuenzahl von Astropecten aranciacus in den Stichprobenfeldern (Ordinate) in bezug auf (a) Tiefe, (b) Sandkorndurchmesser (häufigste Klasse)

den wir A. aranciacus auf groblörnigem bis mittelfeinem Sand; auf solchen Böden stellten wir kleine bis mittelgroße Seesterne fest; auf feinem Sand fehlte $A$. aranciacus oder war selten und trat dann vor allem in großen Exemplaren auf.

Provisorisch knüpfen wir an die Befunde die Überlegungen, daß grober Sand von den großen, kannibalischen Artvertretern gemieden wird und daher ein Refugium für kleinere Seesterne bildet.

Nachdem feststeht, daß $A$, aranciacus mehrere Mollusken-Arten als Beute annimmt, war ein positiver Beitrag der Variabeln „Anzahl Mollusken-Arten“ zur Abundanz von $A$. aranciacus (Abb. 2c) zu erwarten. Die Beziehung dürfte gegenseitig sein: Wo viele Molluskenarten leben, findet $A$. aranciacus ein reichhaltiges Nahrungsangebot und wird abundant; wo $A$. aranciacus kontrollierend wirksam ist, vermehrt sich die Diversität der Beutetier-Arten (PAINE, 1966). 

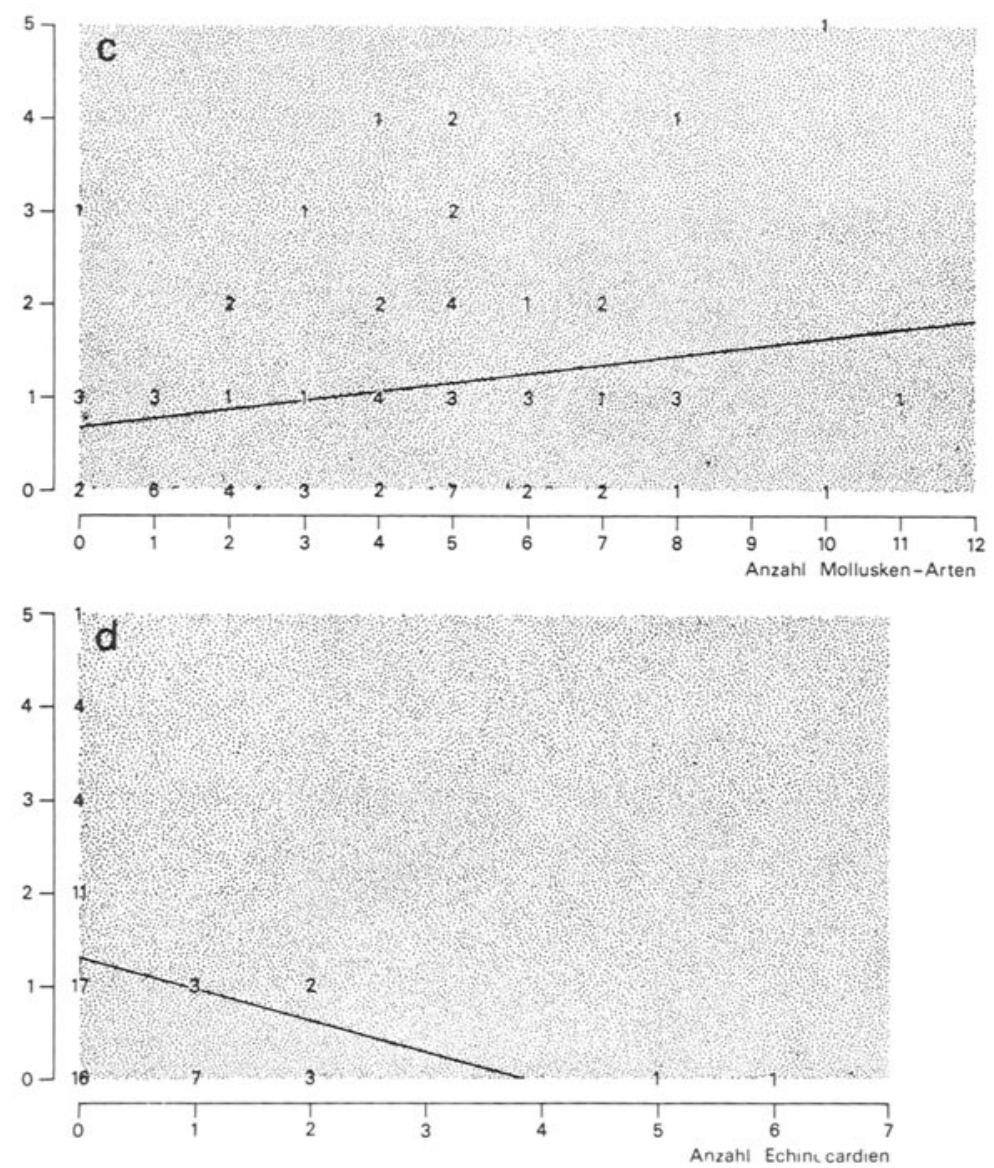

Abb. $2 c$ und d: Einfache lineare Regression der Individuenzahl yon Astropecten aranciacus in den Stichprobenfeldern (Ordinate) in bezug auf (c) Anzahl an Mollusken-Arten und (d) Anzahl an Echinocardien

Aus Magenproben wissen wir, daß Echinocardium ein wichtiges Beutetier von $A$. aranciacus ist. Nun zeigt sich (Tab. 4, Abb. 2d), daß der Faktor „Echinocardium " negativ mit der Abundanz von $A$. aranciacus korreliert ist. Die Regressionsrechnung ist in diesem Fall aber problematisch, da 14 der 31 gefundenen Echinocardien von einer einzigen Tauchstelle in Ufernähe stammen, wo wir A. aranciacus nicht fanden. Biologisch deuten wir den Befund wie folgt: Wo die Seesterne in größerer Dichte auftreten, sind fast alle Echinocardien gefressen worden.

Gesamthaft erklären 13 Kalendertage, die im dummy-System zusammen mit 21 anderen Tagen in die provisorische Regressionsrechnung einbezogen worden waren, $35 \%$ der Variation von A. aranciacus, ein großer Beitrag, der von keinem anderen, einzelnen Faktor erreicht wurde. In der Variablen "Kalendertage" kommen die Gegebenheiten der an einem bestimmten Tag gewählten Beobachtungsorte zur Geltung. 
Außerdem sind verschiedene, nicht ortsgebundene Effekte pauschal enthalten: Wetter, Wasserbewegung und die von ihr bewirkte Wassertribung, Leistungsfähigkeit der Taucher, möglicher Einfluß der Mondphase. Alle diese Effekte mögen einzeln oder interaktiv wirksam sein. Wie schon in der Einleitung dargelegt, verzichten wir darauf, diese Variable zu analysieren, zumal sie sich nur über eine Spanne von 26 Tagen erstreckte.

\section{Nebenergebnisse}

Die Korrelationsmatrix (Tab. 2) enthält mehrere Koeffizienten, die gesichert gröBer als Null sind (bei $5 \%$ Irrtumswahrscheinlichkeit beträgt der kritische Wert 0,232).

\section{Tabelle 5}

Koeffizienten für partielle Korrelation zwischen je zwei unabhängigen biotischen Variabeln. Konstant gehalten wurden die in Tabelle 1 mit Nr. 9 bis 15 bezeichneten abiotischen Variabeln. Aus Versehen wurden "Tageszeit" und „Bucht" nicht eliminiert

\begin{tabular}{|rrrrrrrrrr|}
\hline 1 & 1 & & & & & & & & \\
2 & 0,23 & 1 & & & & & & \\
3 & $-0,14$ & 0,05 & 1 & & & & & \\
4 & 0,10 & 0,45 & $-0,02$ & 1 & & & & \\
5 & $-0,12$ & 0,23 & 0,37 & 0,04 & 1 & & & \\
6 & 0 & 0,14 & 0,16 & 0,04 & 0,27 & 1 & & \\
7 & $-0,04$ & 0,13 & $-0,22$ & 0,10 & 0,14 & $-0,22$ & 1 & \\
8 & 0,21 & $-0,02$ & $-0,24$ & $-0,05$ & $-0,21$ & $-0,03$ & $-0,07$ & 1 \\
\hline & 1 & 2 & 3 & 4 & 5 & 6 & 7 & 8 \\
\hline
\end{tabular}

Somit stoßen wir auf Beziehungen, die der Beachtung wert sind. Allerdings vermindern sich die Koeffizienten, wenn partielle Korrelationen gerechnet werden. Tabelle 5 enthält die Koeffizienten aus partiellen Korrelationen für jede Kombination aus zwei Tierformen, wobei die als anorganisch bezeichneten Variablen eliminiert sind. Die höchsten Korrelationen haben unter diesen Umständen die Anzahl Mollusken-Arten mit der Anzahl Schnecken-Individuen sowie mit der Anzahl Muschel-Individuen, ein Befund, der besagt, daß die Diversität mit der Anzahl Tiere zunimmt. Ferner ist die Anzahl Sipunculida positiv korreliert mit der Anzahl anderer Würmer, Echinocardien, Muscheln und Molluskenarten. Unsere Deutung lautet, daß einige Stellen bei ganz verschiedenen Tiergruppen übereinstimmend große Abundanzen zulassen, auf anderen Stellen die Tierabundanzen allgemein klein sind. Schließlich ist die Anzahl Krebse negativ korreliert mit der Anzahl Echinocardien. Wegen der kleinen Gesamtzahl der Krebse und der lokalen Häufung der Echinocardien möchten wir dieser Korrelation keine Beachtung schenken.

Von den 70 Stichprobenfeldern lagen 28 in weiten Sandflächen, 38 in einem aus Posidonienfeldern und Sandflächen gemischten Gebiet und 4 auf kleinen Sandinseln inmitten weiter Posidonienfelder. Die Eigenschaft wurde als "Flächengliederung" bezeichnet, die Zustände als "offen", "gegliedert" und „geschlossen". Tabelle 6 zeigt für drei Variable, daß ihre Verteilung von der Flächengliederung abzuhängen scheint. So 
Tabelle 6

Die Flächengliederungen "offen“, "gegliedert" und "geschlossen“ wurden in 28, 38 beziehungsweise 4 Stichprobenfeldern registriert. Mittels eines Chiquadrat-Tests wurde untersucht, ob sich die Abundanzen einzelner Tierarten in den drei Flächengliederungen ebenfalls verhalten wie $28: 38: 4$. Hier wiedergegeben sind die drei Fälle, in denen sich ein großer Testwert $\left(\mathrm{X}^{2}\right)$ ergab; der Testwert ist zu vergleichen mit $\chi^{2}$ bei 2 Freiheitsgraden

\begin{tabular}{|c|c|c|c|c|c|}
\hline Tiere & offen & $\begin{array}{l}\text { lächengliede } \\
\text { gegliedert }\end{array}$ & $\begin{array}{l}\text { ing } \\
\text { geschlossen }\end{array}$ & Summe & $X^{2}$ \\
\hline Echinocardium sp. & 24 & 7 & 0 & 31 & 18,36 \\
\hline Würmer & 200 & 174 & 29 & 403 & 20,05 \\
\hline Astropecten aranciacus & 18 & 57 & 2 & 77 & 12,16 \\
\hline
\end{tabular}

fanden sich Echinocardien und Würmer häufiger auf offenen Sandflächen, A. aranciacus häufiger auf gegliederten, als nach Flächengliederung zu erwarten war; fünf weitere Variable (Einsiedlerkrebse, ibrige Krebse, Sipunculida, Schnecken und Molluskenarten) ergaben nur kleine Testwerte. Die Klassifikation nach Flächengliederung bringt erneut zum Ausdruck, daß $A$. aranciacus und Echinocardium negativ assoziiert

Tabelle 7

In den Stichproben wurden 26 Arten Bivalvia, 11 Arten Gastropoda und je 4 Arten Echinodermen und Decapoden gefunden. Die gesiebte Fläche betrug total $17,5 \mathrm{~m}^{2}$

\begin{tabular}{|c|c|c|c|}
\hline Species & $\begin{array}{c}\text { Anzahl } \\
\text { Individuen }\end{array}$ & Species & $\begin{array}{c}\text { Anzahl } \\
\text { Individuen }\end{array}$ \\
\hline Bivalvia & & Gastropoda & \\
\hline Divaricella divaricata & 118 & Natica guillemini & 12 \\
\hline Loripes lacteus & 97 & Natica alderi & 10 \\
\hline Tellina donacina & 91 & Nassa neritea & 10 \\
\hline Modiola adriatica & 41 & Calyptraea chinensis & 6 \\
\hline Diplodonta trigonula & 29 & Chiton sp. & 2 \\
\hline Cardium tuberculatum & 24 & Natica millepunctata & 2 \\
\hline Corbula gibba & 21 & Natica intricata & 1 \\
\hline Donax wariegatus & 21 & Natica baebrea & 1 \\
\hline Thracia papyracea & 19 & Natica josephina & $\hat{1}$ \\
\hline Gonldia minima & 17 & Ceritbium mediterranea & 1 \\
\hline Tellina incarnata & 16 & Scala sp. & 1 \\
\hline Venus gallinat & 12 & seatat sp. & 1 \\
\hline Corbula mediterranea & 12 & & \\
\hline Limatula subauriculata & 11 & Echinodermata & \\
\hline Tellina taenuis & 10 & Echinocardium sp. & 32 \\
\hline Ensis ensis & 8 & Echinocyamus pusillus & 5 \\
\hline Dosina lupinus & 8 & Opbiopsila annulosa & 4 \\
\hline Dosina exoleta & 7 & Holothurie (juv.) & 2 \\
\hline Codakia decussata & 6 & & \\
\hline Tapes aureus & 4 & & \\
\hline Laevicardium crassum & 3 & Decapoda & \\
\hline Gari faroensis & 2 & Portunus depurator & 1 \\
\hline Glycimeris sp. & 2 & Lambrus angulifrons & 1 \\
\hline Pitar rudis & 1 & Diogenes pugilator & 37 \\
\hline Solenomya togata & 1 & Paguristes oculatus & 1 \\
\hline Clamys sp. & 1 & & \\
\hline
\end{tabular}


sind. Berechnet man die partielle Korrelation zwischen zwei Variablen unter Ausschluß aller anderen, bleibt Ecbinocardium korreliert mit offener Flächengliederung.

Von den berücksichtigten Tierformen erwiesen sich die meisten als ungleichmäßig über die beiden Buchten verteilt, wobei kaum zwei Formen dasselbe räumliche Verteilungsmuster erkennen lassen. Wir verstehen den Befund, wenn wir uns die Muster der Abundanzverteilung denken als Folge gegenseitiger trophischer Beziehungen direkter und indirekter - und als Reaktion auf andere, zum Teil chemisch-physikalische Bedingungen, die ebenfalls von Stelle zu Stelle variieren. Welche Bedingungen es sind, die bestimmte Abundanzen beeinflussen, ist kaum zu erkennen. Unsere Methoden decken Abundanz"' und Beziehungsmuster auf, deuten sie aber nicht kausal. Das kausale Verständnis kommt aus anderen Quellen: aus der Kenntnis von Lebensgewohnheiten der Tiere, einschließlich Konkurrenz, Räuber-Beutebeziehung, Dispersionsmechanismen, und so fort. Wünschen wir in der Deutung bessere Gewißheit statt vage Vermutung, müssen wir die Stichprobenmethode extensiv anwenden - sie liefert zwar nie anderes als Indizien -, oder zum Experiment übergehen.

In Tabelle 7 sind die Mollusken, Echinodermen und Decapoden aufgeführt, die beim Sieben von insgesamt $17,5 \mathrm{~m}^{2}$ Fläche erfaßt wurden. Tabelle 8 gibt die Größenverteilung der häufigsten Arten. Aus den Daten lassen sich folgende Schlüsse ziehen: (a) Die Artenzusammensetzung fügt sich in keine der bisher beschriebenen Coenosen ein. (b) Die Bestandesdichte der Mollusken ist mit 36 Individuen pro $\mathrm{m}^{2}$ gering. Doerjes (1971) und Massé (1972) stellen in anderen Gebieten des westlichen Mittelmeeres für einige Muschelarten Dichten fest, die bis zu zwei Größenklassen höher lagen. (c) Mit Ausnahme von Tellina donacina wurden bei den Muscheln fast durchwegs kleine Individuen gefunden, die wir als jung auffassen. (d) Divaricella divaricata und Loripes lacteus kamen gehäuft an einer Stelle mit feinem Sand vor. (e) Tellina donacina wurde in wenigen Exemplaren an fast allen Stellen gefunden. (f) Modiola adriatica war typisch für Stellen mit grobem Sand.

\section{DISKUSSION}

Die Frage lautet, ob Umweltkomponenten, belebte und unbelebte, nachweislich Vorkommen und Abundanz des Seesterns Astropecten aranciacus beeinflussen. Bei den belebten Komponenten, mit denen zu rechnen ist, wurde von Feinden abgesehen, und da $A$. aranciacus im untersuchten Gebiet die weitaus häufigste Seestern-Art war, konnten auch Konkurrenten außer Betracht fallen. Es blieb zu prüfen, ob Vorkommen und Abundanz von Beutetieren von Einfluß seien. Beim begrenzten Umfang, den die Arbeit unter Wasser hatte, ließen sich nur zwei Beziehungen dieser Art nachweisen. Eine einfache negative Korrelation zwischen den Anzahlen von $A$. aranciacus und Echinocardium verkleinerte sich, als partielle Korrelationen gerechnet wurden, und kam auch in der multiplen Regression nur schwach zum Ausdruck. Nun ist aber $A$. aranciacus als Räuber, der die Infauna bejagt, auf das Vorhandensein von Beutetieren angewiesen; insofern bedarf der magere Befund einer Interpretation. Vermutlich ist das Jagdrevier des rasch umherstreifenden Räubers (FERLIN, 1973) groß im Vergleich zu der Fläche, die von Aggregaten der zumeist kleinen Beutetiere eingenommen werden. Auch 
Vorkommen von Astropecten aranciacus

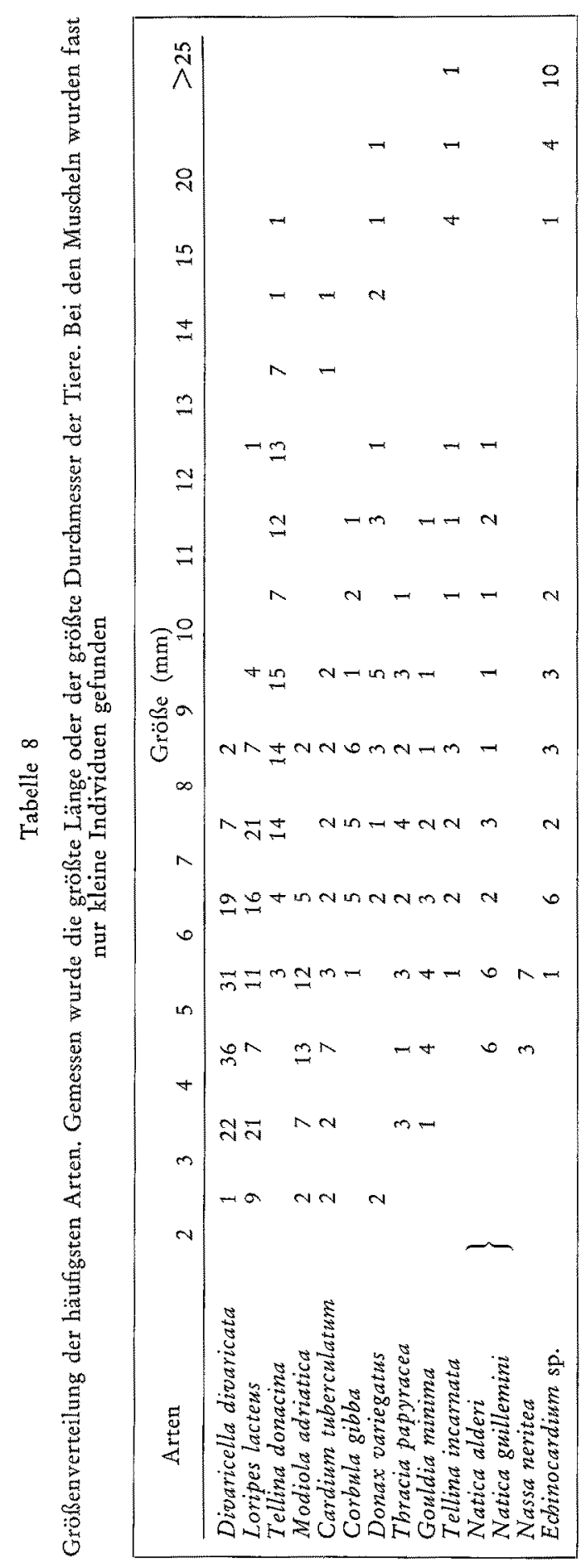


lassen die Nebenergebnisse erkennen, daß verschiedene Beutetierarten unabhängig voneinander aggregieren, so daß sich im Jagdrevier von $A$. aranciacus die Zusammensetzung der Beutetierfauna über kurze Strecken ändert. Die Annahme, das Untersuchungsgebiet sei besetzt von einer einheitlichen Lebensgemeinschaft im Sinne von PiCARD (1965) und $A$. aranciacus gehöre ihr an, entspräche nicht der Realität. Wir schließen nicht aus, daß $A$. aranciacus da verharrt und sich ansammelt, wo gute Fanggründe liegen. Wenn aber eine Konzentration von Beutetieren $A$. aranciacus da und dort abundanter werden läßt als anderswo, können es an jeder Stelle verschiedene Beute-Arten sein, oder Gesellschaften, die in Form von "noda " in wechselnder Zusammensetzung und Ausdehnung vorkommen (Amouroux, 1972). Im übrigen sind Astropecten-Arten bekannt für ein breites Nahrungsspektrum (Hulings \& HEMLAY, 1963; Christensen, 1970; Wells et al., 1961; KIsCH, 1958), was auch für $A$. aranciacus gilt (MAssé, 1975, und eigene unveröffentlichte Beobachtungen).

Von den physikalischen Faktoren ( $z u$ denen auch das Vegetationsmerkmal zu zählen ist, da es lediglich die Umwelt strukturiert) beeinflussen die Sedimenteigenschaften das Vorkommen von $A$. aranciacus. TorTonese (1948) registrierte $A$. aranciacus auf den verschiedensten Sedimenten, häufiger jedoch auf grobem Sand als auf Schlamm. Nach Parker (1975) gehören Sedimenteigenschaften zu den wesentlichsten abiotischen Faktoren, die in seinem Untersuchungsgebiet die räumliche Verteilung benthaler Lebensgemeinschaften bestimmen. DoERJEs (1971) vermutet, daß die Tiefenzonierung der Venus-gallina-Cönose von Sedimenteigenschaften abhängt, wobei allerdings das Sediment dem Einfluß von Wassertiefe und Wellengang an der Küste unterliegt. Bei koexistierenden Räubern rechnet KoHN (1971) damit, daß sie sich nicht nur auf die Nahrung, sondern auch auf ein Substrat spezialisieren können, und er zitiert CHARTock, der bei Schlangensternen eine aktive Präferenz für eine Substratsorte feststellte. In ähnlicher Weise (allerdings lokal ohne Konkurrenten) dürfte die Häufigkeit des Seesterns $A$. aranciacus von einer physikalisch definierbaren Sedimentsorte abhängen. Es kann sein, daB $A$. aranciacus sinnesphysiologisch und verhaltensökologisch auf ein bestimmtes Sediment geprägt ist. Im Endeffekt wichtig ist dann der trophische Vorteil, der ihm aus dieser Bindung erwächst. Tatsächlich unterscheiden sich die Sedimentsorten im Gehalt an Organismen (Marzolf, 1965; STanley \& James, 1971; Amouroux, 1972). Unsere Deutung: Indem sich $A$. aranciacus nach der Sedimentstruktur orientiert, wird er abundant an Stellen, die für ihn trophisch günstig sind.

Daß bei $A$. aranciacus die Tiefe von Einfluß ist, war ebenfalls zu erwarten, kommen doch viele Arten des Benthals zoniert vor (MAssé, 1966; GILAT, 1964; WAHL, 1971; Amouroux, 1972; und andere). Im Untersuchungsgebiet fällt der Meerbodeu mit konstanter Neigung gegen die Tiefe $a b$, und die Tiefenzone von 3 bis $10 \mathrm{~m}$, in der $A$. aranciacus abundant ist, bildet ein relativ schmales Band. Seine untere Grenze stimmt überein mit dem Beginn der kompakten Posidonienwiesen, in die der Seestern nicht eindringt (in benachbarten Buchten, wo die Posidonienwiesen erst in größerer Tiefe beginnen, kommt $A$. aranciacus auch unterhalb $10 \mathrm{~m}$ Tiefe vor). Dagegen ist an seiner oberen Grenze des Vorkommens auf dem Meerboden keine Grenzlinie zu sehen. Es ist denkbar, daß $A$. aranciacus die Wassertiefe direkt oder indirekt perzipiert und die zu geringe Tiefe meidet.

In der Literatur finden sich nur wenige Angaben über die Bestandesdichte von 
Astropectiniden im Mittelmeer. Massé (1975) stellte an der Küste der Provence eine maximale Dichte von einem $A$, aranciacus auf $23 \mathrm{~m}^{2}$ fest. Die mittlere Dichte schätzte er auf ein Tier je $50 \mathrm{~m}^{2}$; sie ist etwa halb so groß wie die von uns ermittelte.

Wegen ihrer Größe und ihres Räubertums können adulte Astropectinidae nur in geringer Dichte vorkommen, was von vornherein eine Stichprobenentnahme mit dem Peterson-Greifer oder ähnlichen Hilfsmitteln unwirksam erscheinen läßt. Sogar die Zählung der sichtbaren, aktiven Tiere durch Taucher ergibt nur die relative Dichte, da stets ein Teil der Population unsichtbar im Sand vergraben liegt. Auf diese Schwierigkeiten dürfte es zurückzuführen sein, daß in faunistischen oder biozönologischen Arbeiten (DoERJEs, 1971; GILAT, 1964) Astropectiniden nur am Rande erwähnt sind, obwohl ihnen im Sandbiotop eine ökologisch bedeutungsvolle Rolle zukommt.

\section{ZUSAMMENFASSUNG}

1. Astropecten aranciacus L. ist die größte von sechs Arten der Gattung, die im Mittelmeer nachgewiesen sind. Dieser Seestern lebt auf Sedimentböden; die obere Grenze des Vorkommens findet sich auf etwa drei Meter Tiefe.

2. Auf 70 Stichprobenfeldern von je $25 \mathrm{~m}^{2}$ Fläche wurde die Individuenzahl von $A$. aranciacus zusammen mit biotischen und abiotischen Bedingungen registriert. Das Untersuchungsgebiet lag $\mathrm{z}$ wischen 3 und $10 \mathrm{~m}$ Tiefe.

3. Die statistische Datenauswertung ergab, daß die Abundanz von $A$. aranciacus mit zunehmender Tiefe und steigendem mittlerem Sandkorndurchmesser zunimmt. Von den biotischen Faktoren war die Anzahl Molluskenarten positiv, die Anzahl Echinocardien negativ mit der Abundanz von A. aranciacus korreliert.

4. Die Bestandesdichte von $A$, aranciacus schwankte in dem heterogenen, mosaikartig gegliederten Biotop zwischen 0 und 12 Individuen je $100 \mathrm{~m}^{2}$. Im Mittel entfielen auf $100 \mathrm{~m}^{2} 4$ Seesterne. Der Radius $R$ der Tiere variierte $z$ wischen 7 und $15 \mathrm{~cm}$.

5. Die Makro-Infauna des Gebiets fiel durch geringe Abundanz auf. Bei den Mollusken betrug die durchschnittliche Bestandesdichte 36 Tiere pro $\mathrm{m}^{2}$. Dagegen war die Arten-Diversität groß: In Infaunenproben von gesamthaft $17,5 \mathrm{~m}^{2}$ Fläche fanden sich 37 Molluskenarten.

Danksagungen. Die Aufenthalte am Meer wurden ermöglicht durch Beiträge der Carl Hescheler-Stiftung und der Georges und Antoine Claraz-Schenkung. Mehrere Tauchkollegen halfen beim Sammeln der Daten. Bei der Bestimmung der Infauna war Dr. J.-M. Amouroux, Banyuls-sur-Mer, Frankreich, behilflich. V. LubINI-Ferlrn analysierte die Sandproben unter Beratung von Dr. B. BLASER in der Eidgenössischen Anstalt für Forstliches Versuchswesen. Frau C. Hesse-Honegger zeichnete die Abbildungen.

\section{ZITIERTE LITERATUR}

Amouroux, J.-M., 1972. Donnés sur la structure et l'instabilité des peuplements infralittoraux de la côte du Roussillon. Thèse, Université de Paris, $192 \mathrm{pp}$.

Burla, H., Ferlin, V., Pabst, B. \& Rrbi, G., 1972. Notes on the ecology of Astropecten aranciacus. Mar. Biol. 14, 235-241. 
Cherbonnier, G., 1958: Edhinodermes. Faune mar. d. Pyrén.-orient. 2, 1-67.

Christensen, A. M., 1970. Feeding biology of the sea-star Astropecten irregularis Pennant. Ophelia 8, 1-134.

Doer Jes, J., 1971. Der Golf von Gaeta (Tyrrhenisches Meer). IV. Das Makrobenthos und seine küstenparallele Zonierung. Senckenberg. marit. 3, 185-201.

Draper, N. R. \& Smith, H., 1966. Applied Regression Analysis. Wiley, New York, 407 pp.

FerLIN, V., 1973. The mode of dislocation of Astropecten aranciacus. Helgoländer wiss. Meeresunters. 24, 151-156.

GilAT, E., 1964. The macrobenthonic invertebrate communities on the mediterranean continental shelf of Israel. Bull. Inst. océanogr. Monaco 62 (1290), 1-46.

Hulings, N. C. \& Hemlay, D. W., 1963. An investigation of the feeding habits of two species of sea-stars. Bull. mar. Sci. Gulf Caribb. 13, 354-359.

KIsch, B. S., 1958. Astropecten irregularis precieux auxiliaire du Malacologiste. Bull. Cent. Etud. Rech. scient. Biarritz 2, 9-15.

KOEHLER, R., 1921. Echinodermes. Faune Fr. 1, 1-210.

KoHN, A. J., 1971. Diversity, utilization of resources, and adaptive radiation in shallow-water marine invertebrates of tropical oceanic islands. Limnol. Oceanogr. 16, 422-436.

Marzolf, G. R., 1965. Vertical migration of Pontoporeia affinis (Amphipoda) in Lake Michigan. Great Lakes Research Division, University of Michigan, Publ. No. 13, 1-133.

Massé, H., 1966. Contribution à l'écologie du genre Astropecten Linck. Red Trav. Stn. mar. Endoume 41, 187-191.

- 1972. Quantitative investigations of sand-bottom macrofauna along the Mediterranean North-West Coast. Mar. Biol. 15, 209-220.

- 1975a. Etude de l'alimentation de Atsropecten aranciacus Linné. Cah. Biol. mar. 16, 495-510.

- 1975b. Ethologie alimentaire de Astropecten aranciacus L. In: 9th European marine biology sympositum. Ed. by H. Barnes. Aberdeen Univ. Press, Aberdeen, 343-355.

PaINE, R. T., 1966. Food web complexity and species diversity. Am. Nat. 100, 1-65.

PARKER, R. H., 1975. The study of benthic communities. Elsevier, Amsterdam, 279 pp.

PiCARD, J., 1965. Recherches qualitatives sur les biocoenoses marines des substrats meubles dragables de la région Marseillaise. Recl. Trav. Stn. mar. Endoume 52, 1-160.

Stanley, D. J. \& James, N. P., 1971. Distribution of Echinarachnius parma (Lamarck) and associated fauna on Sable Island Bank, Southeast Canada. Smithson. Contr. Earth Sci. 6, $1-24$.

SurTs, B., 1957. Use of dummy variables in regression equations. J. Am. statist. Ass. 52, 548-551.

Tortonese, E., 1948. Variazioni fenotipiche e biologia della populazione di Astropecten aranciacus (Echinodermi) del Golfo di Napoli, con riferimenti a specie congeneri. Boll. Mus, zool. Univ. Torino 1, 87-123.

- 1965. Echinodermata. Fauna Ital. 6, 1-422.

VAHL, O., 1971. Growth and density of Patina pellucida (Gastropoda: Prosobranchia) on Laminaria hyperborea (GunNERUS) from Western-Norway. Ophelia 9, 31-50.

Wells, H. W., Wells, M. J. \& Gray, I. E., 1961. Food of the sea-star Astropecten articulatus. Biol. Bull. mar. biol. Lab., Woods Hole 120, 265-271.

Anschrift des erstgenannten Autors: Prof. Dr. H. BurlA

Zoologisches Museum

Künstlergasse 16

CH-8006 Zürich

Schweiz 